BNI 40666

BNL- -40666

DE88 005605

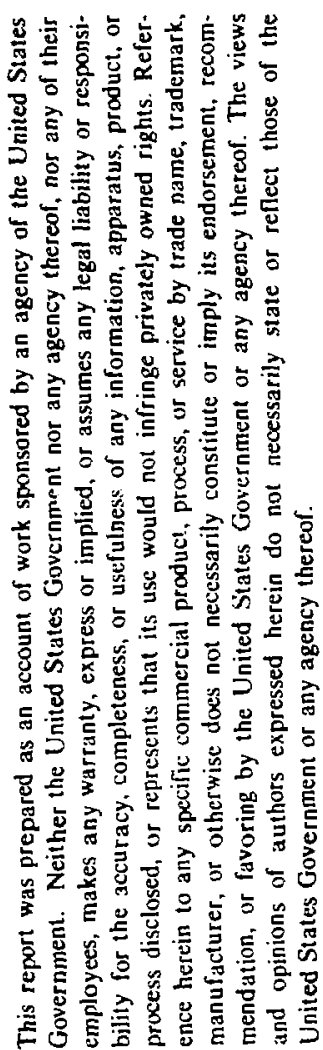

\title{
A HIGH INTENSITY POSITRON BEAM AT THE BROOKHAVEN
}

\section{REACTOR}

M. Weber," K.G. Lynn," Lo. Roellig."

A.P. Mills, Jr, ${ }^{3)}$ A.R. Moodenbaugh,

1) Ciry College of the City University of New York, NY 10031.

2) Brookhaven National Laboratory, Upron, NY 11973.

3) A.T.\&.T. Bell Laboratories, Murray, NJ 07974.

\section{ABSTRACT}

We describe a high intensity, low energy positron beam utilizing high specific activity ${ }^{64} \mathrm{Cu}$ sources $(870 \mathrm{Ci} / \mathrm{g})$ produced in a reactor with high thermal neutron Aux. Fast-to-slow moderation can be performed in a self moderation mode or with a transmirsion moderator. Slow positron rates up to $1.6 \times 10^{8} \mathrm{c}^{+} / \mathrm{s}$ with a half life of $12.8 \mathrm{~h}$ are calculated. Up to $1.0 \times 10^{8} \mathrm{e}^{+} / \mathrm{s}$ have been observed. New developments including a $\mathrm{Ne}$ moderator and an on-line isotope separation process are discussed.

\section{INTRODUCTION}

A number of proposed positron experiments require a high intensity slow $e^{-}$beam to attain an acceptable signal to noise ratio.' Such a beam could be used for differential cross section measurements of positrons scattering in gases. ${ }^{2}$ The $2 \gamma$ angular correlation measurements of the Fermi surfaces of metals have begun to be exiended co surfaces of solids. ${ }^{3}$ The beam has been used as a source for a monoenergetic positronium beam to study the possibility of positronium scattering and diffraction from surfaces." If this proves to be practical, such a new tool would greatly supplement results oa neutral atom beams." A high positron count rate would also make feasible rare decay studies, such as the measurement of the single phocon decay rate of positrons. ${ }^{6}$ The implementation of several stages of remoderation, 2 technique that recently has been realized for the first time, could provide a microscopic beam usable as a positson microprobe or to study matter-antimatter systems at high densities.'

At present two ways to reach these high count rates exist and are being further developed. In one approach the bremsstrahlung of a $100 \mathrm{MeV}$ electron beam of a LINAC hitting a target produces showers of positron-electron pairs. ${ }^{9}$ The positrons are moderated to form the low energy beam. Due to the nature of the LINAC, a pulsed beam of positrons is available.

\section{MASTER}


The second method, described here, uses thermal newtrous to produce the positron soterce ${ }^{64} \mathrm{Cu}$. A reactor with a high thermal neutron flux such as the Hight Flux Beam Reactor (HFBBR) al Brookhaven National Laboratory (BNL) is essemtial. If available as a single crystal, the copper can be used as the moderator, a combination, that we call a self moderator. Alternately the positrons can be modetated by a thin single crystal film; a tungsten film has been used successfully as such a transmission moderator. ${ }^{10.1}$

The reacior based beam will be discussed in more detail below. Section 2 contains calculations to predict the performance of the beam, given the available neutron flux. Self moderation and iransmission moderation are compared. In section 3, advantages and disadvantages of sucli a beam are presented. The technical realization of such a beam at BNL is described in section 4. Problems that were encountered during beam development are presented in section 5 . The present beam performance, as well as improvements planned or under way, are covered. Section 6 provides an outlook, and an appendix contains the detailed self moderator calculations outlined in section 3 . Also the possibility of further increasing the count rate by an on-line enrichment of ${ }^{64} \mathrm{Cu}$ is considered. ${ }^{12}$ Finally the ongoing implementation of the recently developed neon moderator is discussed. ${ }^{13}$

\section{CALCULATIONS TO PREDICT THE BEAM PERFORMANCE}

Production of the Positron Activity

The source of positrons, ${ }^{G} \mathrm{Cu}$, is used. It is produced in the High Flux Beam Reactor (HFBR) at Brookhaven by means of the themal neutron reaction ${ }^{63} \mathrm{Cu}(\mathrm{n}, \gamma)$ ${ }^{64} \mathrm{Cu}$. A flux of $\mathrm{f}=8.3 \times 10^{14} \mathrm{n}_{\mathrm{o}} / \mathrm{cm}^{2} \mathrm{~s}$ of thermal neutrons ${ }^{14}$ near the core, along with the relative high cross section of $\sigma=4.5$ b for this reaction, make reasonable amounts of ${ }^{64} \mathrm{Cu}$ possible. The short half life of ${ }^{64} \mathrm{Cu}$ of $\mathrm{t}_{1 / 2}=12.8 \mathrm{~h}$ necessitales frequent renewal of the source.

With a production rate $\lambda_{\mathrm{f}}=\mathrm{f} \sigma$ and decay ratc $\lambda_{\mathrm{d}}=\ln 2 / \mathrm{t}_{1 / 2}$ after an irradiation of time I the positron activity is

$$
A(i)=\operatorname{lp} \frac{m}{M} N_{a} \lambda_{p}\left[1-e^{-\lambda_{d} d}\right]
$$

This formula holds for the case $\lambda_{p} \ll \lambda_{d}$.

In the equation $b=19 \%$ is the fraction of decays through the positron channel, $p$ the fraction of ${ }^{63} \mathrm{Cu}$ in a sample of mass $\mathrm{m}$, and $\mathrm{M}=63.54 \mathrm{~g} / \mathrm{mol}$ the molar weight. $N_{a}$ is Avogadro's number.

After an irtadiation or duration

$$
t_{m}=\frac{1}{\lambda_{d}} \ln \frac{\lambda_{d}}{\lambda_{p}}
$$

the maximal activity is reached. Figure 1 shows the activity $A(1) / A\left(t_{m}\right)$ as a function of the irradiation time. For the neutron flux available at the HFBR the $95 \%$ of $\wedge\left(t_{m}\right)$ is 
r. ached after - 55 hours. Figure 2 shows the dependence of the activily on the neutron nux on a $\log$-log scale. Irradiation times are chosen 10 maximize the activity in each case.

\section{Performance of Copper as a Moderator}

A system of $\mathrm{Cu}(111)$ on $\mathrm{W}(110)$ with a perfect interface is considered. The efficiency $\varepsilon$, defined as the ratio of slow positrons to fast positrons, of such a moderator with the source as an iniegral part of the copper layer is made up of several contributions. These are the efficiencies resulting from a single crystal of copper on a

\section{Copper source strength}



Fig. 1. Fraction of the maximum activity of a copper sample, as a function of the irradiation time in the reactor. The thermal neutron flux is $8.3 \times 10^{14} \mathrm{no}_{0} / \mathrm{cm}^{2} \mathrm{~s}$.

\section{Copper source strength}

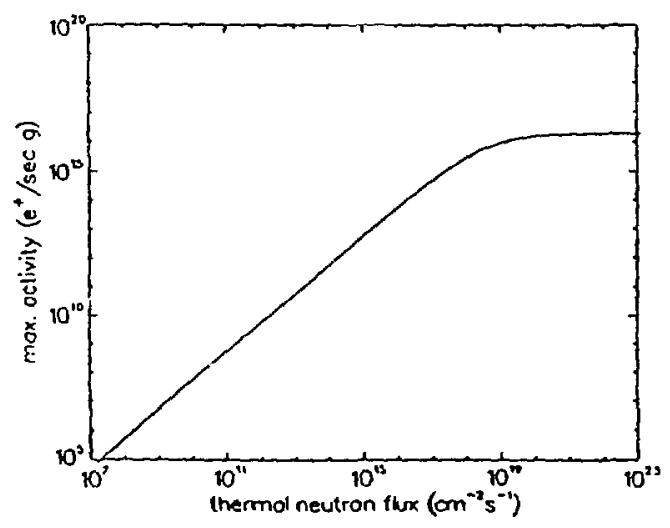

Fig. 2. Maximum positron activity of a sample that can be reached depending on the thermal neutron flux. $99 \%$ entiched ${ }^{63} \mathrm{Cu}$ is used 
tungsten substrate, the backscantering effect of the tungsten, and the moderation capability of the tungsten crystal.

These contributions are calculated in the appendix and are shown in figure 3. When the $\mathrm{Cu}$ layer is on the order of $10^{3} \mathrm{~nm}$ thick or more only the contribution of the $C u$ layer itself remains significant. $\varepsilon_{a}$ can be approximated by

$$
\varepsilon_{s}(d)=\frac{1}{2} Y_{0} \frac{L_{+}}{d}\left[L_{+} \alpha \ln \frac{1+L_{+} \alpha}{1-L_{+} \alpha}+\int_{0}^{1} d t \frac{1}{t-L_{+} \alpha}\left(1-e^{-\alpha d / t}\right)\right] \text {. }
$$

The fraction diffusing back from the $\mathrm{W}$ substrate is

$$
\varepsilon_{w}(d)=\frac{1}{2} Y_{0} \frac{L_{+}}{d}(1-f) \frac{L_{w} \alpha_{w}}{L_{+} \alpha} \frac{1}{\cosh d / L_{+}} \int_{0}^{1} d t \frac{t}{t+L_{w} \alpha_{w}}\left(1-e^{-\alpha d / t}\right) .
$$

Here $d$ is the thickness of the copper layer, $\alpha$ and $\alpha_{w}$ are the products of mass absorption coefficient and density $p$ of copper and tungsten respectively, ${ }^{7.12} \mathrm{f}$ is the backscatter fraction for positrons from the $\beta^{+}$-spectrum off the W-Cu interface, $L_{+}$and $L_{w}$ are the diffusion lengths for positrons in copper and cungsten, and $Y_{0}$ is the branching ratio $\left[Y_{0}=0.55\right.$ for $\left.\mathrm{Cu}(111)+S\right]$.

\section{Performance with a W(100) Transmission Moderator}

An alternative to self moderation is the recently developed transmission moderator. ${ }^{10.11}$ It consists of a thin $\left(<10^{3} \mathrm{~nm}\right)$ single crystal of tusgsten. Its moderation

\section{Copper Selfmoderator}

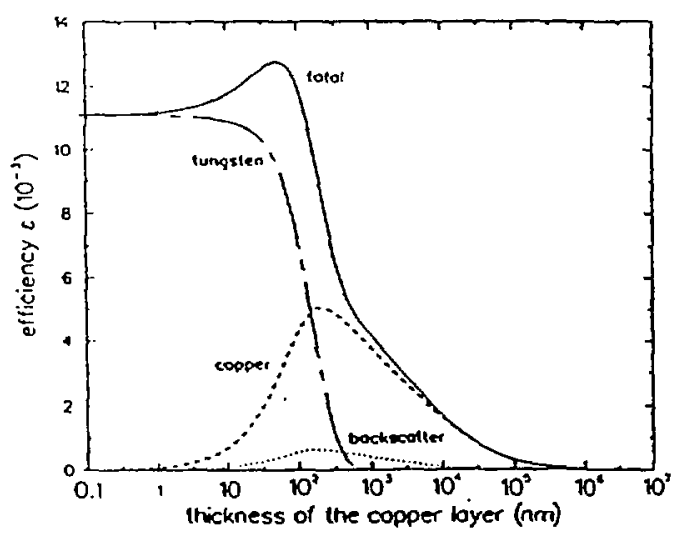

Fig. 3. The various contributions to the efficiency $\varepsilon$ of the reactor positron source. . . . the single crystal $\mathrm{Cu}$ !ayer, ..... the fraction due to backscattering off the interface, _ _ _ _ the $W$ substrate, and the toral efficiency. 
efficiency is theoretically estimated to be $c_{0}=4 \times 10^{-3}$ (emitted slow positrons per fast positrons hitting the crystal). ${ }^{10}$ Due to self absorption of fast positrons in the source, its activity is reduced by a factor $s$.

$$
s(d)=\frac{1}{\alpha d} \int_{0}^{1} d t \quad\left[1-e^{-a d t}\right] .
$$

Further losses depend on the source-moderator geometry factor $\mathbf{G}$. $\mathbf{G}$ is at most 0.5 since only one side of the source faces the moderator. The absolute efficiency with this type of moderation would then be

$$
\varepsilon_{1}(d)=G s(d) \varepsilon_{0} .
$$

\section{Expected Performance of the Reactor Beam}

For a discussion of a self moderator, the efficiency as the fraction of the available activity that contributes to the beam is not very useful. With growing thickness of the crystal the efficiency will decrease but this will be more than compensated by the larger amount of available activity.

In the appendix the product

$$
E=\varepsilon d
$$

is introduced. Figure 4 shows the various components of $E$ again as a function of the thickness of the Cu layer and constant specific activity. Beyond a $10^{5} \mathrm{~nm}$ thick copper film significant gains in $E$ are no longer possible. At this thickness only the copper contribution is significant. The source-transmission moderator geometry and the value $E_{0}$ determine which type of moderation is superior. The efficiency of a tungsten single crystal foil was estimated in reference 10 to be $\varepsilon_{0}=4 \times 10^{-3}$ for a $10^{3} \mathrm{~nm}$ thick crystal. In figure $S$ the performances of both moderators are shown, $G$ is assumed to be 0.25 . Ir should be noted that the best reported efficiency for a transmission moderator is $5 \times 10^{-4}$.

These values present an upper limit. The thermal neutron flux decreases during a reactor cycle (at BNL about 5\%). The capsule absorbs neutrons (<5\%). The beam becomes operational about 2 hours after the new source is removed from the reactor, in which time the copper has decayed to about $90 \%$ of its original strength.

A typical source of $100 \mathrm{mg}$ is irradiated for 48 hours. A $10^{5} \mathrm{~nm}$ thick copper crystal of about $1 \mathrm{~cm}^{2}$ area is produced. It has a positron-activity of $A=14.9 \mathrm{Ci}$ of positrons. Self moderation is calculated to deliver a beam of $A=4.3 \mathrm{mCic} / \mathrm{s}$ $=1.6 \times 10^{8} \mathrm{e}^{+} / \mathrm{s}$ and a transmission moderator $\mathrm{A}=2.5 \mathrm{mCie} \mathrm{C}^{+}=9.3 \times 10^{7} \mathrm{e}^{+} / \mathrm{s}$ with $\varepsilon_{1}=10^{-3}$.

\section{DISCUSSION OF THE HFBR BEAM}

The high positron activity of the source material is achieved by utilizing the reactor's high thermal neutron flux of $8.3 \times 10^{14} n_{\mathrm{d}} / \mathrm{cm}^{2} \mathrm{~s}$. A $(\mathrm{n}, \gamma)$ reaction converts 


\section{Copper Selfmoderator}

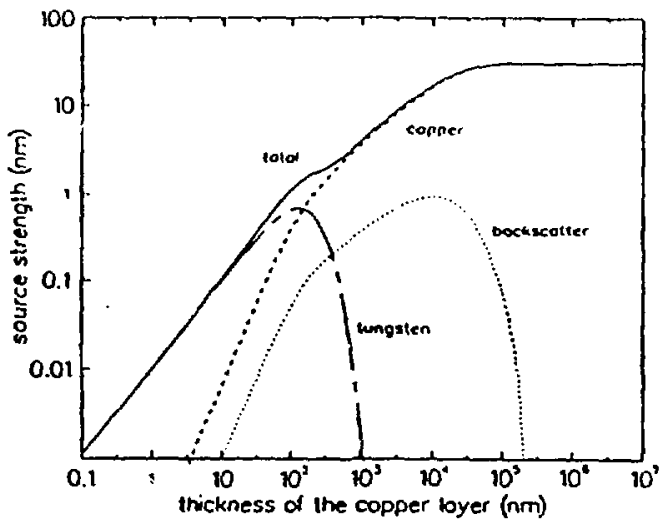

Fig. 4. The source strength on a logarithmic scale as a function of the thickness of the copper layer. The same symbols as in fig. 3 are used.


Fig. 5. Comparison of the efficiency $\varepsilon$ of a self moderating film of $\mathrm{Cu}$ (dashes) and a transmission moderator when located in front of the $\mathrm{Cu}$ film (dots).

${ }^{63} \mathrm{Cu}$ into the $12.8 \mathrm{~h}$ half life positron emitter ${ }^{64} \mathrm{Cu}$. The fraction of $69.1 \%$ of usable ${ }^{63} \mathrm{Cu}$ occurring in natural copper is usually increased to about $99 \%$ in enriched copper. Thus positron activities of $166 \mathrm{Cie} / \mathrm{g}$ can be achieved with 48 hour irradiations. After irradiation, the copper is transferred into the vacuum system, and evaporated onto a W(110) crystal surface. On this surface the epitaxial growth of single crystal copper in the (111) direction is preferred. ${ }^{16} \mathrm{Cu}(111)$ is the most suitable orientation if used as a moderator. ${ }^{17}$ In this case the copper will, be the source as well as the moderator. 
Periodic irradiation, of sources does not imternupt or disturb work performed by other reactor users. The only interference with a steady supply of new sources are infrequent sample irradiations by other users of this high flux reactor por. In such cases a port with a somewhat lower neutron flux can be used. In contrast most LINACs cannot suppon the positron beam facility and other experiments simultaneously.

The short half life of the source is advantageous when maintenance work has to be done in the source chamber. In addition to the source material, only a few radioactive isotopes are produced. By using high purity copper, the radioactivity of impurities is kept more than six orders of magnitude below that of the ${ }^{64} \mathrm{Cu}$ isotope. At a LINAC the bremsstrahlung causes a high background radiation and activales material in the vicinity of the positron source. While positrons from pair production are unpolarized, a beam of polarized positrons is possible when they originate in $\boldsymbol{\beta}^{+}$decays as in ${ }^{54} \mathrm{Cu}$.

The reactor-based beam operates in a pseudo DC mode that avoids the problem of pileup of signals during the short bursts of positrons in a pulsed beam. A pulsed beam is advantageous for time of flight experiments.

\section{THE TECHNICAL REALIZATION}

The Reactor Division routinely irradiates samples for all kinds of research. They have chosen aluminum capsules as containers that are insened through thimbles into the reactor core at the end of long aluminum tubes. The apparatus to transfer the copper pellet from the reactor into the UHV system was based on this design. The copper sources produced by the reactor cannot be handled directly. The radiation level is high enough to require a remote control system for the transfer of sources into the vacuum chamber. Thie chamber must be shielded. A concrete house, referred to as the blockhouse, has been constructed around the source chamber. It is capable of protecting against up to $10 \mathrm{kCi}$ sources.

The capsule is held in a lead pig for about one hour to let the shor-lived isotopes decay. It is cus off the Al tube and drops from the lead container on top of the blockhouse, through a drying chamber into a shear mechanism, where the source pellet is removed. In the drying chamber the small amounts of tritium contaminated cooling water from the reactor thimble on the capsule are evaporated and blown through filters in the reactors decontamination facility. Passing an airlock, the copper pellet reaches the crucible, where it is evaporated onto a tungsten crystal and forns the position source.

In the shear the capsule is broken open to release the source pellet. The sliearing process tears Aakes off the capsule wall, which can fall into the crucible along with the pellet. These would poison the moderator crystal and greatly reduce the positron intensity of the beam. To prevent this, the part of the capsule that is sheared is made of high purity copper. The empty capsule and the plug are dumped into a lead pig inside the blockhouse.

As it drops out of the airlock, the copper pellet is guided through a tube into an alumina crucible. The copper is evaporated onto a $W(110)$ crystal, which induces the crystalline growth of $\mathrm{Cu}(111)$. Annealing and a subsequent $\mathrm{H}_{2} \mathrm{~S}$ treatment increase the moderator efficiency of the copper. Depending on the initial evaporation, annealing improves the moderator performance by one order of magnitude while the sulfur treatment may gain another $20 \%$. An $x$-ray study of a copper film verified the $\mathrm{Cu}(111)$ 
structure. 13y resistive heating of the tungsten crystal, the copper can be evaporated onto a dump after it has decayed and the tungsten crystal is ready for a new source. Afier several source cycles the dump is replaced to keep the accumulation of radioactive material low.

An overview of the beam line is shown in figure 6. In the "run" position the copper-coated tungsten faces the beam line. The crystal can be c:ectrically floated to give the beam its transport energy. An accelerator tube in front of this crystal position improves the geometry of the electric field. Here a tungsten single crystal transmission moderator of about $10^{3} \mathrm{~nm}$ thickness can be rotated into place. A location close to the source is important to improve the geometry on which the efficiency depends to a large extent.

The slow positrons are transported by a magnetic guiding field. ${ }^{18.19}$ Two sets of $\vec{E} \times \vec{B}$ plates guide the beam around a $\gamma$-ray shield and energy filter the positrons. The positrons can be guided to one of two experimental areas. A set of apertures on one line permits a continuous acceleration up io $16 \mathrm{keV}$. The other line is equipped with diffusion pumps and a large turbo pump to differentially pump a gas cell where. 2 positronium (Ps) beam is produced.

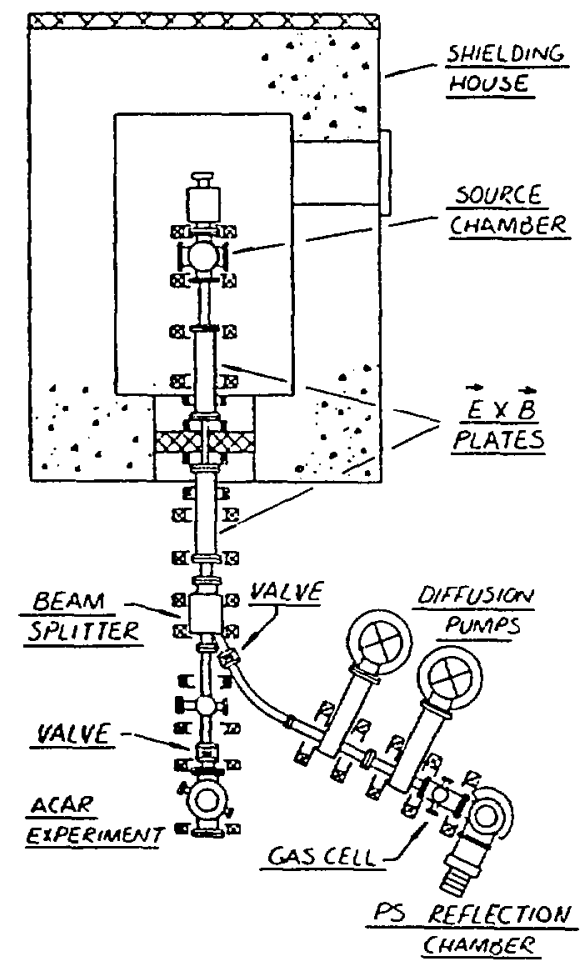

Fig. 6. Top view of the positron beam at the Brookhaven Reactor. Slow positrons are extracted from the source chamber and magnetically guided through two $\vec{E} \times \vec{B}$-regions out of the blockhouse. In the splitter the positrons can be directed to two experimental areas, the ACAR chamber or the Ps-beam line (PS). Shut off valves permit a separation from the main beam line. 
As the source material, both spectroscopic pure copper metal $(69.1 \%$ of usable ${ }^{6.1} \mathrm{Cu}$ ) or isotopic ${ }^{63} \mathrm{Cu}$ oxide (about $99 \%$ of ${ }^{63} \mathrm{Cu}$ ) from Oak Ridge National Laluoratory (ORNL) can be utilized. The isotopic copjer oxide is first reduced to metal. Care must le taken to keep the copper free of impuritics that would inlibit its use as a good self mnderator.

\section{PROBLEMS, IMPROVEMENTS AND RESULTS}

Iron and cobalt impurities in the capsule material caused higher than expected radiation levels, owing to neutcon induced activation. Higher purity aluminum is now used as the capsule material. The capsule walls are kept thin to accumulate less radioactive waste.

Impurities and dirt that were transferred with the copper poisoned the moderator. Capsules now have a copper piece where they are sheared. Much of the copper was lost during evaporations from an open wire basket. Now an outgassed alumina insen in the wire basket increases the copper yield on the tungsten. Also, the contamination of the vacuun chamber with copper is greally reduced.

Of prime concern are impurities that are already contained in the source material. An analysis of the $\gamma$-ray spectrum of an irradiated pellet showed lines of several metals. Only high grade speciroscopic copper or carefully enriched and reduced material can be used. ${ }^{G 4} \mathrm{Cu}$ also decays $10{ }^{64} \mathrm{Zn}$ via a $\beta^{-}$-decay, Some of this ${ }^{64} \mathrm{Zn}$ will be converted to ${ }^{65} \mathrm{Zn}$ which emits a $1.115 \mathrm{MeV} \gamma$-ray during its decay with a hallive of 245 days. Although the activity of impurities is below $1 \mathrm{mCi} / \mathrm{g}$ of copper they accumulate to significant amounts due to the longer half lives. For this reason, the copper dump is changed periodically. The evaporation procedure is still being refuned to produce copper films with a better crystal structure and fewer defects.

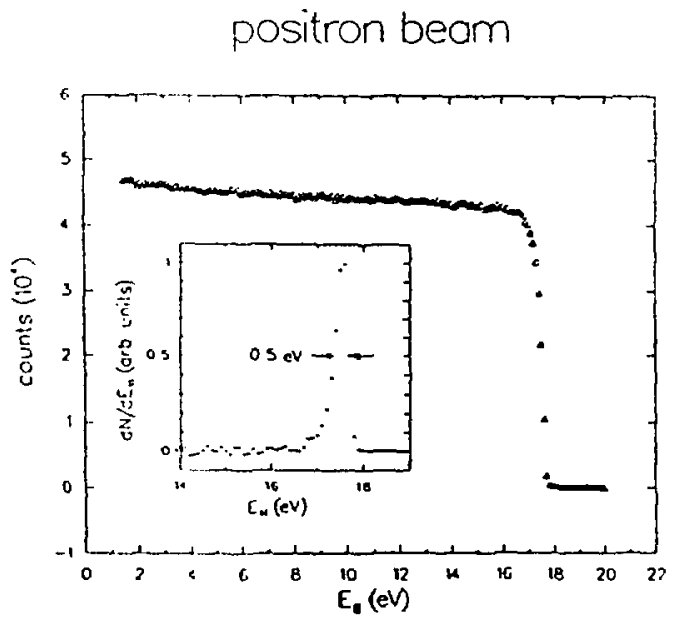

Fig. 7. Retarding potential measurement of the longitudinal energy spread of tie positzon beam at $18 \mathrm{eV}$ energy. The retarding potential was applied on the gas cell at the end of the Ps-beam line. 
Count rates up $101 \times 10^{8} \mathrm{e}^{4} / \mathrm{s}$ have been reacived using the self moderation mode. "The transmission moderator produced lower rates and it was dismantled as the source evaproration becane more reliable. In the gas cell, after the curve in the bean line, the IFWI the energy spread of the beam was measured to be on the order of $0.5 \mathrm{cV}$ (see figure 7).

\section{OUTLOOK}

In order to make measurements and estimates of the conversion efriciency of the moderator possible, a surface barrier detector will be installed to count fast $\beta$-particles coming off the source. A neon moderator will be tested and will finally replace the copper self moderator. The implementation is discussed in the appendix.

The possibility of mass-separation of ${ }^{64} \mathrm{Cu}$ from the much more abundant but inactive ${ }^{61} \mathrm{Cu}$ is being investigated. About $2.4 \times 10^{-4}$ of the copper atoms are ${ }^{64} \mathrm{Cu}$ after a 48 hour irradiation. An improvement of this ratio by a mass separation during the evaporation will increase the specific activity. The source crystal could be made thinner. A higher efficiency $\varepsilon$ (see fig. 3 ) will result in a higher count rate of positrons in the beam. This also will be discussed in the appendix.

A hybrid guiding system is envisioned for a second generation system. The positrons are extracted electrostatically from the source region. For longer distances a magnetic guiding field is planned, which returns to an electrostatic system at the experimental area if required. The source chamber could be floated up to $100 \mathrm{keV}$. Finally several beam switches are planned to make this beam a multi-user facility.

\section{APPENDIX}

The calculation of the efficiency of a self moderator provides the expressions used in section 3. More detailed estimates about the planned neon moderator are presented in the following part. An estimate of possible improvements of the self moderator by incorporating an on-line separation system of ${ }^{64} \mathrm{Cu}$ into the system concludes the appendix.

\section{The self moderator}

Positrons origirating in $\beta^{+}$-decays of the ${ }^{64} \mathrm{Cu}$ thermalize rapidly and diffuse throughout the crystal. Either they annihilate with electrons or reach the surface. If they do not fall into a surface state or pick up an electron and form Ps they are expelled into the vacuum due to the negative workfunction of the crystal. $N$. difications to this part result from the fact that the source material is not a free stan wing foil. The substrate - $W(110)$ in this case - causes a change in the boundary conditions at the interface side. A fraction $f$ of fast positrons will be reflected back into the copper layer. The remaining positrons penetrate the interface and thermalize in the tungsten. They all diffuse throughout the material and have a finite probability of returning to the surface where they can contribute to the positron beam. Since the workfunction of tungsten is more negative than the workfunction for copper this junction acts like a diode which tends to push the thermal positrons our of the tungsten.

The average time for positrons to thermalize $(<10 \mathrm{ps})$ is very shon compared to the half life of the source. Consequently the steady state solution of the diffusion equation adequately describes the situation. In addition the large area of the source moderator in relation to its thickness permits a one dimensional calculation. A perfect interface is assumed in this derivation. Further the possibility of positrons scattering 
out of the source while they thermalize parallel to and near the surface is neglected. These assumptions end to overestimate the resulting $\mathfrak{c}$.

From the exponential implantation profile of a source far reenoved from the moderator the profile for a source moderator can be calculated. Figure 8 illustraies how the reduction to one dimension has been performed. The exponential profile leads to the source moderator profile

$$
P_{s}(x)=\frac{1}{d} \int_{0}^{t} d t\left[1-e^{-\frac{1}{2} \alpha d / 1} \cosh \frac{\alpha(d / 2-x)}{t}\right] ; t \leq \cos \theta .
$$

Alpha can be calculated from the empirical formula ${ }^{15}$

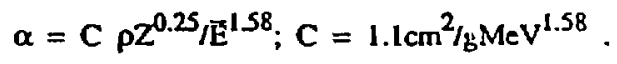

$d$ is the thickness of the crystal. $\rho$ is the density, $Z$ the atomic number and $E$ the mean energy of the $\beta^{+}$-spectrum ( $E=0.2 \mathrm{MeV}$ for copper). Similarly the contributions to the implantation profile due to the back reflection $P_{f}(x)$, and the implantation into the tungsten $P_{w}(x)$ are calculated. Figure 9 shows the various implantation profiles.

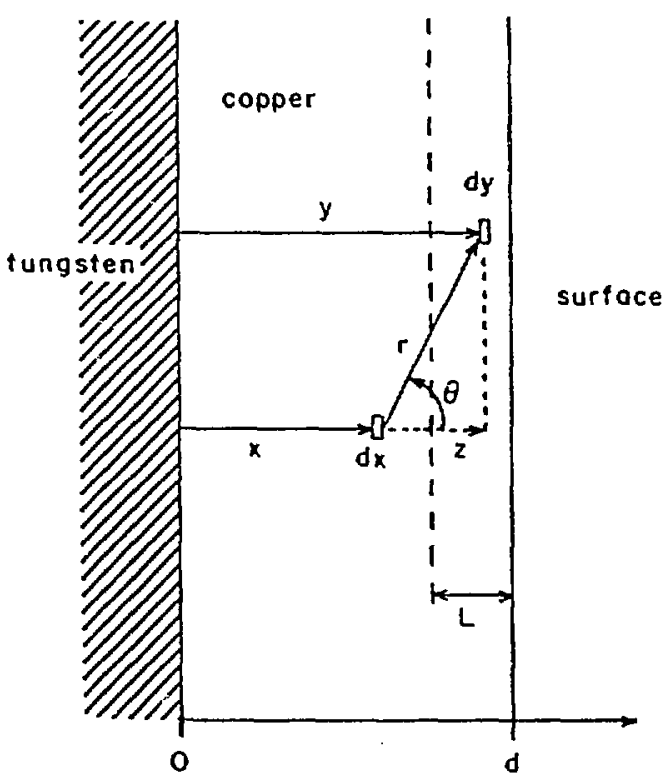

Fig. 8. Schematic to illustrate the calculation of the 1-dimensional implantation profiles. The positrons created at $\mathbf{d x}$ stop after a flight path $\mathbf{r}$ in the direction $\theta$ at dy. Integration across the source yields the implantation profile. The source area is assumed to be large compared to its thickness. 


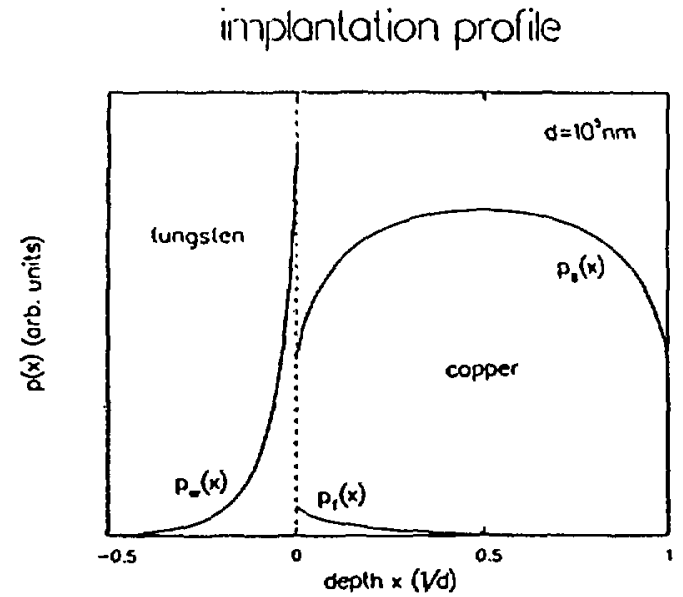

Fig. 9. The contributions to the implantation profile of the $\mathrm{Cu}$ self moderator on a single crystal $W$ substrate. Shown are the profile from the self moderating source $P_{S}(x)$, the fraction due to backscattering $P_{f}(x)$, and the implantation into the $W$ substrate $P_{w}(x)$. The $C u$ layer is $10^{3} \mathrm{~nm}$ thick.

$$
\begin{aligned}
& p_{r}(x)=\frac{1}{2 d} f \int_{0}^{l} d t\left(1-e^{-\alpha d / t}\right) e^{-\alpha w / t} \\
& p_{w}(x)=\frac{1}{2 d}(1-f) \frac{\alpha_{w}}{\alpha} \int_{0}^{i} d t\left(1-e^{-\alpha d / t}\right) e^{+\alpha_{w} x / t}
\end{aligned}
$$

The implantation profiles are used in the diffusion equation for a steady state. The density $n(x)$ of positrons vanishes at the copper surface and deep in the tungsten. The flux $j(x)$ is continuous at the interface.

The fraction $Y_{0}$ is emitted as slow positrons. $Y_{0}$ and $j(d)$ form the efficiency of the self moderator $\varepsilon(d)$. The various contributions amount to

$$
\begin{aligned}
\varepsilon_{s}(d)= & \frac{1}{2} Y_{0} \frac{L_{+}}{d}\left[L_{+} \alpha \int_{0}^{l} d t \frac{t}{t^{2}-L_{+}^{2} \alpha^{2}}\left(1-e^{-\alpha d t}\right)\right. \\
& +\tanh \frac{d}{L_{+}}\left[L_{+} \alpha \ln \frac{1+L_{+} \alpha}{1-L_{+} \alpha}+\int_{0}^{1} d t \frac{t^{2}}{t^{2}-L_{+}^{2} \alpha^{2}}\left(1-e^{-\alpha d / t}\right)\right) \\
+ & \left.\frac{1}{\cosh \frac{d}{L_{+}}} L_{+} \alpha \int_{0}^{t} d t \frac{t}{t^{2}-L_{+}^{2} \alpha^{2}}\left(1-e^{-\alpha d t}\right)\right]
\end{aligned}
$$




$$
\begin{aligned}
& \varepsilon_{d}(d)=\frac{1}{2} Y_{0} \frac{L_{+}}{d} f\left[L_{+} \alpha \int_{0}^{1} d t \frac{1}{t^{2}-L_{+}^{2} \alpha^{2}}\left(1-e^{-\alpha d t}\right) e^{-\alpha d t}\right. \\
& +\tanh \frac{d}{L_{+}} \int_{0}^{t} d t \frac{t^{2}}{t^{2}-L_{+}^{2} \alpha^{2}}\left(1-e^{-\alpha d t}\right) e^{-\alpha d t} \\
& 106 \\
& \left.-\frac{1}{\cosh \frac{d}{L_{+}}} L_{+} \alpha \int_{0}^{1} d t \frac{1}{t^{2}-L_{+}^{2} \alpha^{2}}\left(1-e^{-\alpha d t}\right)\right] \\
& \varepsilon_{w}(d)=\frac{1}{2} Y_{0} \frac{L_{t}}{d}(1-f) \frac{L_{w} \alpha_{w}}{L_{+} \alpha} \frac{1}{\cosh \frac{d}{L_{+}}} \int_{0}^{1} d t \frac{t}{t+L_{w} \alpha_{w}}\left(1-e^{-\alpha d t}\right) \quad 10 c
\end{aligned}
$$

Figure 3 shows the efficiency of a crystal depending on its thickness $d$. For copper $Y_{0}=0.55$ and $f=0.17$ was estimated for the $C u-W$ interface. ${ }^{20}$ With growing thickness $\varepsilon(d)$ decreases. On the other hand this is more than compensated by the larger amount of source activity. In the case of a source moderator the product $E(d)$ of efficiency and thickness describes the situation better.

$$
E(d)=d \varepsilon(d)
$$

7

The absolute source strength is the product of the specific source activity, the source area, and $E(d)$. Figure 4 shows the various parts of $E$ on a logarithmic scale versus the thickness of the copper layer. At about $10^{5} \mathrm{~nm} E$ levels off. Additional amounts of copper do not improve the source strength unless the area is increased.

For $d$ of about an order of magnitude larger than $L_{+}=110 \mathrm{~nm}$

$$
\tanh \frac{d}{L_{+}}=1 ; \cosh \frac{d}{L_{+}}<10^{-4} \text {. }
$$

which simplifies equations (10) and (7) to

$$
E(d)=\frac{1}{2} Y_{0} L_{+}\left[L_{+} \alpha \ln \frac{1+L_{+} \alpha}{1-L_{+} \alpha}+\int_{0}^{1} d t \frac{1}{1-L_{+} \alpha}\left(1-e^{-\alpha d t}\right)\right]
$$

11 
The Neon moderator

As mentioned by Mills and Gullikson rare gas solids and solid neon in panicular, can be used as positron moderators. ${ }^{13}$ They report a high yield $\left(Y_{n}=0.7\right)$ and a large diffusion length $\left(L_{+} \approx 10^{3} \mathrm{~nm}\right)$ for solid neon. A moderation efficiency $\varepsilon=7 \times 10^{-3}$ measured by them is higher then any previously known moderator. Neon was condensated onto a cylindrical source geometry to take advantage of the large reflection coefficient of Ne for slow positrons.

The first neon moderated beam ${ }^{21}$ performed at the high initial level over a period much larger than the half life of ${ }^{64} \mathrm{Cu}$. With the cylindrical source geometry self absorption of a ${ }^{64} \mathrm{Cu}$ source can be reduced. The larger surface area of the cylinder permits a thinner copper layer without increasing the beam cross section. The efficiency of a copper source-neon moderator combination was estimated using the same approach as for ahe efficiency calculation for a self moderator presented in this paper. A $3000 \mathrm{~nm}$ thick layer of $\mathrm{Ne}$ on a $1.6 \times 10^{4} \mathrm{~nm}$ thick Cu source result in an efficiency $\varepsilon=0.64 \%$. A $100 \mathrm{mg}$ source of enriched ${ }^{63} \mathrm{Cu}$ irradiated for two days and evaporated onto a $7 \mathrm{~cm}^{2}$ area of a cup ( $1 \mathrm{~cm}$ diam.; $2 \mathrm{~cm}$ high) would yield close to $4 \times 10^{9} / \mathrm{sec}$ slow positrons.

The reported energy spread of $0.58 \mathrm{eV}$ for neon moderators is acceptable. Some advantages of the neon moderator are as follows: 1) no single crystal zupper is necessary; 2) no spectroscopically pure material is required; 3) the copper does not have to be removed prior to a new source evaporation. The cup itself can be replaced after several months. The design of a liquid He cooling system inside a $\mathrm{LN}_{2}$ cooled thermal shield to achieve a temperature of $6 \mathrm{~K}$ appears to be straight forward. Possible disadvantages are that the base pressure of the vacuum systen should be in the $10^{-10}$ tor range to prevent contamintion and the excellent properties of the neon moderator may be affected by the high radiation. level of the source. A neon moderator for the reactor beam will be insialled in the near future.

\section{On-line Isotope Separation}

The plan is to vaporize the copper and then selectively ionize the ${ }^{64} \mathrm{Cu}$ atoms and only collect shese on the tungsten substrate. The ionization could be carried out by means of tunable dye lasers. This method would take advantage of the different hyperfine structure splitting of the atomic energy levels of the various copper isotopes.

At about $200 \mathrm{~nm}$ thickness the efficiency of a self moderator peaks. (see fig 3) A reasonable compromise between source size and positron rate is a layer of this thickness. A present about 1 in $4000{ }^{63} \mathrm{Cu}$ atoms will be converned to ${ }^{64} \mathrm{Cu}$ during one irradiation. The proposed separation process enhances the ratio of ${ }^{64} \mathrm{Cu}$ to ${ }^{63} \mathrm{Cu}$ by a factor of 1000 . Then up to $24 \%$ of the source will be active. The total efficiency at $200 \mathrm{~nm}$ thickness is about $5 \times 10^{-3}$ and the result would be a beam of $1 \times 10^{10} \mathrm{e} / \mathrm{s}$ with a $1 \mathrm{~cm}^{2}$ source area. To achieve this it would be necessary to irradiate $=280 \mathrm{mg}$ of isotopic copper for 3 days.

The separation requires some time. A rate of $10^{-8} \mathrm{~g}{ }^{64} \mathrm{Cu} / \mathrm{min}$ was estimated. Then evaporation times of $2-3$ hours are necessary. On the other hand the area of the source could be reduced without losses in brightness and within a shorter irradiation time on the order of an hour. A beam of several $\mathrm{nm}^{2}$ size and $10^{8} \mathrm{e}^{+} / \mathrm{s}$ seems to be realistic. 


\section{ACKNOWLEDGEMENTS}

The authors would like to thank J. Zahradka for his design effors and J. Rutherford and P. Schnitzenbaumer for their technical help. J. Hurst provided the capsules with the pellets Work of D. Rorer and T. Holmquist, was essential for the success of the project. D. M. Chen's effor with the transmission moderator is very much appreciated. This work is supported in part by the Division of Materials Sciences, U.S. Dept. of Energy, under Contract No. DE-AC02-76CH00016, and in part by the National Science Foundation through Grant No. DMR-8315691.

\section{REFERENCES}

1. For example: A.P. Mills, Jr., in "Positron scattering in gases," J.W. Humberston and M.R.C. McDowell eds. Plenum Press, 121 (1984).

2. R.J. Drachman in "Positron Scattering in Gases," ibid., p. 121.

3. R.H. Howell, P. Meyer, I.J. Rosenberg, J.J. Fluss, Phys. Rev. Lett 54, 1698 (1985) and K.G. Lynn, A.P. Mills, Jr., R.N. West, S. Berko, K.F. Canter, L.O. Roellig; Phys. Rev. Lett. 54, 1702 (1985).

4. To be presented by $L$. Roellig in these proceedings.

5. For example: H. Hoinkes, Rev. Mod. Phys. 52, 933 (1980) and refs. therein.

6. K.G. Lynn, D.N. Lowy, I.K. McKenzie, J. Phys. C: Solid State Phys. 13, 919 (1980).

7. A.P. Mills, Jr., Appl. Phys. Lett. 23, 189 (1980).

8. W.E. Frieze, D.W. Gidley, K.G. Lynn, Phys. Rev. B31, 5628 (1985).

9. R.H. Howell, R.A. Alvarez in "Positron Scattering in Gases," as 1., p. 155.

10. A. Vehanen, J. Mäkinen, Appl. Phys. A 36, 97 (1985).

11. D.M. Chen, K.G. Lynn, R. Pareja, B. Nielsen, Phys. Rev. B 31, 4133 (1985).

12. R. Engleman, Jr., R.A. Keller, C.M. Miller, N.S. Nogar, F.A. Paisner, Nucl. Inst. and Meth. in Phys. Res. Section B 26, 448 (1987)

13. E.M. Gullikson, A.P. Mills, Jr., Phys. Rev. Lett. 57, 376 (1986).

14. In "HFBR Handbook," S. Shapiro, D.C. Rorer, H. Kuper eds., informal report Brookhaven National Laboratory.

15. M. Mourino, H. Löbl, R. Paulin, Phys. Lett. 71A, 106 (1979).

16. A. Vehanen, K.G. Lynn, P.J. Schultz, M. Eldrup, Appl. Phys. A 32, 163 (1983).

17. A.P. Mills, Jr. in Proc. LXXXIII Intern. School of Physics "Enrico Fermi", W. Brandt, A. Dupasquier eds. (Academic Press, New York 1982).

18. A.P. Mills, Jr., P.M. Platzman, B.L. Brown, Phys. Rev. Lett. 41, 1079 (1978).

19. A.P. Mills, Jr., Phys. Rev. Lett. 41, 1828 (1978).

20. H.E. Hansen, S. Linderoth, K. Petersen, Appl. Phys. 229, 99 (1982).

21. A.P. Mills, Jr., E.M. Gullikson, Appl. Phys. Lett. 49, 1121 (1986). 